\title{
Effect of exercise on distribution of pulmonary blood flow in patients with mitral stenosis
}

\author{
J. Bjure, ${ }^{1}$ B. Liander, and J. Widimský \\ From the Department of Clinical Physiology and Medical Clinic I, Sahlgrenska sjukhuset, \\ University of Göteborg, Sweden; and Institute for Cardiovascular Research, Praha-Krc, \\ Czechoslovakia
}

The distribution of pulmonary blood flow was studied with the ${ }^{133}$ Xenon technique in 15 patients with mitral valvular disease at rest, and during and after exercise. The central haemodynamics were studied with standard catheterization techniques.

With regard to the distribution of pulmonary blood flow at rest two groups of patients were identified. One had a normal pattern of distribution and the other a decreased relative blood flow. The second group had a significantly higher left atrial mean pressure at rest.

During exercise the pulmonary perfusion tended to become more even. Patients with a high relative basal blood flow decreased this flow during exercise in the same manner as normal subjects. On the other hand, patients with a low relative basal flow increased this flow during exercise, and this increased flow persisted 15 minutes after exercise.

It is assumed that this change in patients with low basal blood flow is due to vasodilatation in basal areas during and after exercise.

The use of the radioactive isotope ${ }^{133}$ Xenon has made it possible to study the distribution of pulmonary blood flow. In healthy subjects this technique has disclosed that in the sitting position a perfusion gradient exists, the basal areas being about three times more perfused than the apical ones (Bake, Bjure and Widimský, 1968; Ball et al., 1962; West and Dollery, 1960; West, 1962). In patients with pulmonary hypertension this perfusion gradient disappears, and in severe mitral stenosis an inverse perfusion pattern may be found (Ball $e t$ al., 1962; Dawson, Kaneko, and McGregor, 1965; West, Dollery, and Heard, 1965). A direct relation between the left atrial pressure and the perfusion gradient has been described (Jebavý et al., 1970). Thus the distribution of pulmonary perfusion might be used as an indirect indicator of the severity of mitral stenosis.

In a previous paper the effect of exercise on the perfusion gradient in sitting healthy subjects was studied. It was found that with exercise the perfusion became more even, i.e. pulmonary blood flow increased more in the apical than in the basal area (Bake et al., 1968).

Received 8 July 1970

1 Address correspondence to: J. Bjure, Department of Clinical Physiology, Sahlgrenska sjukhuset, 413 45 Göteborg, Sweden.
The purpose of this study was to investigate the regional pulmonary perfusion at rest, and during and after moderate exercise in patients with mitral stenosis, with an attempt to relate the changes in perfusion to the clinical status of the patient.

\section{Methods and procedure}

Fifteen patients with rheumatic heart disease (I4 with predominant mitral stenosis and I with mitral insufficiency) were studied.

The equipment and method were the same as those described by Bake et al. (1967). The patients were studied in the sitting position on a bicycle ergometer with detectors placed dorsally, 4 or 5 detectors on each side arranged in a slit collimation system. The most apical detector was positioned about $5 \mathrm{~cm}$ from the top of the lung and the most basal detector about $25 \mathrm{~cm}$ from the top. Injections of $0.5 \mathrm{mCi}^{133}$ Xenon were given during breath holding at the end of the normal expiration through a percutaneously inserted catheter with the tip approximately in the subclavian vein. When the ${ }^{133}$ Xenon had reached the alveoli as judged from rate meters the subjects inspired until a full inspiration was achieved. All ${ }^{133}$ Xenon measurements were performed with held breath at full inspiration. Data from each region were expressed as the percentage of the totally registered activity per unit ventilated lung volume.

Two injections were given when the patients had been sitting 30 minutes on a bicycle ergo- 
TABLE I Clinical data

\begin{tabular}{|c|c|c|c|c|c|c|}
\hline $\begin{array}{l}\text { Case } \\
\text { No. }\end{array}$ & $\begin{array}{l}\text { Age } \\
(y r)\end{array}$ & Sex & Diagnosis & $\begin{array}{l}\text { Functional } \\
\text { group }\end{array}$ & $\begin{array}{l}\text { Vital } \\
\text { capacity }\end{array}$ & $F E V \% \dagger$ \\
\hline $\mathbf{I}$ & 52 & $\mathbf{F}$ & Mitral stenosis and insufficiency & 3 & $2 \cdot 4$ & 83 \\
\hline 2 & 37 & $\mathbf{M}$ & Mitral stenosis and mitral and aortic insufficiency & 2 & $4 \cdot 0$ & 66 \\
\hline 3 & 56 & $\mathbf{F}$ & $\begin{array}{l}\text { Mitral stenosis and insufficiency, restenosis, } \\
\text { opn } 1963\end{array}$ & $3-4$ & $\mathbf{2} \cdot \mathbf{I}$ & 90 \\
\hline 4 & 47 & $\mathbf{M}$ & Mitral stenosis & 2 & $3 \cdot 7$ & 68 \\
\hline 5 & 54 & $\mathbf{F}$ & $\begin{array}{l}\text { Mitral stenosis, and aortic insufficiency; } \\
\text { restenosis, opn } 1957\end{array}$ & 3 & $\mathbf{2 \cdot 2}$ & 73 \\
\hline 6 & 57 & $\mathbf{F}$ & Mitral stenosis & $\mathbf{I}-\mathbf{2}$ & $1 \cdot 9$ & 79 \\
\hline 7 & $7 \mathbf{I}$ & $\mathbf{F}$ & Mitral stenosis & $3-4$ & $1 \cdot 4$ & 83 \\
\hline 8 & 58 & $\mathbf{M}$ & Mitral stenosis and insufficiency, opn 1959 & 3 & $3 \cdot 4$ & 57 \\
\hline 9 & 62 & $\mathbf{F}$ & Mitral stenosis and insufficiency & $3-4$ & $2 \cdot 0$ & 70 \\
\hline Io & 59 & $M$ & Mitral insufficiency & $3-4$ & $3 \cdot 0$ & 87 \\
\hline I I & 54 & $\mathbf{M}$ & Mitral stenosis & 3 & $5 \cdot 1$ & 82 \\
\hline 12 & 60 & $\mathbf{F}$ & Mitral stenosis and mitral and aortic insufficiency & 3 & $3 \cdot \mathbf{I}$ & 74 \\
\hline 13 & 63 & $\mathbf{F}$ & Mitral stenosis and insufficiency & 3 & $2 \cdot 0$ & 83 \\
\hline 14 & 43 & $\mathbf{F}$ & Mitral stenosis & 2 & 3.4 & 79 \\
\hline 15 & 47 & $\mathbf{M}$ & Mitral stenosis & 2 & $4 \cdot 0$ & 83 \\
\hline
\end{tabular}

* Vital capacity in I BTPS.

$+\mathrm{FEV} \%=$ forced expiratory volume in percentage of vital capacity.

meter. After the second injection the patient started to exercise and injections were given after 4 and 7 minutes of exercise. Within 2 minutes and at 15 minutes after exercise the 5 th and 6 th injections were given. The load was adjusted to the functional group of the patients and was between 150 and $450 \mathrm{kpm} / \mathrm{min}$.

Fourteen of the I 5 patients were studied haemodynamically. In 12 patients right heart catheterization was performed and in 13 patients left atrial pressure was measured by the transseptal technique, as used in this laboratory (Paulin and Varnauskas, I962). In one patient pulmonary wedge pressure was used as an indicator of left atrial pressure. A polyethylene catheter was percutaneously introduced into the brachial artery by the Seldinger technique.

Fourteen patients were studied at rest and 9 both at rest and during supine exercise at loads varying from 'no load' to $200 \mathrm{kpm} / \mathrm{min}$. Pressures in the pulmonary artery, left atrium, brachial artery, and right atrium were measured.

Cardiac output was estimated by direct Fick procedure in 2 patients and by dye dilution method using cardiogreen or BSP as indicator in I I patients. All the techniques were the same as earlier described (Forsberg, I964). Pulmonary vascular resistance was calculated according to the formula :

$$
\frac{\overline{\mathrm{P}}_{\mathrm{PA}}-\overline{\mathrm{P}}_{\mathrm{LA}}}{\dot{\mathrm{Q}}} \mathrm{mmHg} /(1 . / \mathrm{min}) \text {. }
$$

\section{Results}

Clinical and laboratory data of the patients are shown in Table 1 . Table 2 shows the haemodynamic data. Tables 3 and 4 show the perfusion data from each region expressed as the percentage of the totally registered activity per unit ventilated lung volume for the right and left lungs respectively.

TABLE 2 Central haemodynamics at rest and during exercise

\begin{tabular}{|c|c|c|c|c|c|c|c|c|}
\hline $\begin{array}{l}\text { Case } \\
\text { No. }\end{array}$ & & $\overline{\mathrm{P}}_{P A}$ & $\overline{\mathbf{P}}_{L A}$ & $\dot{\mathbf{Q}}$ & $S V$ & $P V R$ & $\overline{\mathbf{P}}_{B A}$ & $\overline{\mathrm{P}}_{R A}$ \\
\hline \multirow[t]{2}{*}{ I } & Rest & $3 I$ & I8 & $5 \cdot 5$ & 48 & $2 \cdot 4$ & 98 & I \\
\hline & Exercise 200 & 49 & 23 & $7 \cdot 6$ & 60 & $3 \cdot 4$ & 104 & - \\
\hline \multirow[t]{2}{*}{2} & Rest & 22 & 15 & $6 \cdot 1$ & 92 & $I \cdot I$ & 68 & 5 \\
\hline & Exercise I50 & 44 & $3 I$ & $8 \cdot 2$ & 88 & $I \cdot 6$ & $8 I$ & - \\
\hline \multirow[t]{2}{*}{3} & Rest & - & - & $4 \cdot 3$ & 78 & - & 88 & 7 \\
\hline & Exercise Ioo & - & 15 & $7 \cdot 4$ & 93 & 一 & 一 & I I \\
\hline 4 & Rest & 21 & 16 & 5.0 & 66 & $I \cdot 0$ & 94 & 2 \\
\hline \multirow[t]{2}{*}{5} & Rest & 25 & IO & $2 \cdot 9$ & 43 & $5 \cdot 2$ & 91 & 4 \\
\hline & Exercise I00 & 43 & 35 & - & - & - & 92 & - \\
\hline 6 & - & - & - & 一 & - & - & - & - \\
\hline 7 & Rest & 51 & 27 & $3 \cdot 9$ & 39 & $6 \cdot 2$ & 71 & 7 \\
\hline 8 & Rest & 55 & 26 & $2 \cdot 7$ & 49 & 10.7 & 95 & 8 \\
\hline \multirow[t]{2}{*}{9} & Rest & I5 & 12 & $2 \cdot 2^{\star}$ & 37 & $I \cdot 4$ & 85 & $\mathbf{I}$ \\
\hline & Exercise no load & - & 22 & - & - & - & - & - \\
\hline IO & Rest & 36 & 26 & $4 \cdot 0^{\star}$ & 48 & $2 \cdot 5$ & I I9 & 8 \\
\hline \multirow[t]{2}{*}{ I I } & Rest & 33 & 19 & $5 \cdot 2$ & 81 & $2 \cdot 7$ & 84 & 2 \\
\hline & Exercise I 50 & IO9 & 45 & $7 \cdot 8$ & 60 & $8 \cdot 2$ & 109 & - \\
\hline \multirow[t]{2}{*}{12} & Rest & 35 & 20 & $4 \cdot 6$ & 40 & $3 \cdot 3$ & 86 & 4 \\
\hline & Exercise I 50 & 48 & $3 I$ & 6.9 & $5 I$ & $2 \cdot 5$ & 89 & - \\
\hline I3 & Rest & 27 & Io (PCV) & $3 \cdot 6$ & 58 & $4 \cdot 7$ & 76 & 3 \\
\hline \multirow[t]{2}{*}{14} & Rest & - & 22 & - & - & - & - & - \\
\hline & $\begin{array}{l}\text { Exercise no load } \\
\text { Rest }\end{array}$ & - & 42 & - & $\overline{8}$ & - & - & - \\
\hline \multirow{2}{*}{ I 5} & Exercise 200 & $\begin{array}{l}16 \\
38\end{array}$ & $\begin{array}{l}10 \\
23\end{array}$ & $\begin{array}{l}3 \cdot 4 \\
7 \cdot 3\end{array}$ & $\begin{array}{l}48 \\
7 I\end{array}$ & $\begin{array}{l}I \cdot 8 \\
2 \cdot I\end{array}$ & 92 & 3 \\
\hline & & 20 & 23 & 13 & 11 & $2 \cdot 1$ & & \\
\hline
\end{tabular}

Symbols: $\overline{\mathbf{P}}_{\mathrm{PA}} \quad=$ mean pulmonary artery pressure

$$
\begin{aligned}
& \left.\begin{array}{l}
\overline{\mathrm{P}}_{\mathrm{LA}}=\text { mean left atrial pressure } \\
\overline{\mathrm{P}}_{\mathrm{BA}}=\text { mean brachial artery pressure }
\end{array}\right\} \mathrm{mmHg} \text {. } \\
& \overline{\mathbf{P}}_{\mathrm{RA}}=\text { mean right atrial pressure } \\
& \mathrm{PCV}=\text { pulmonary capillary venous pressure. } \\
& \dot{\mathbf{Q}}=\text { cardiac output, } 1 . / \mathrm{min} \text {. } \\
& \text { SV = stroke volume, } \mathrm{ml} \text {. } \\
& \text { PVR = pulmonary vascular resistance, } 1 . / \mathrm{min} / \mathrm{mmHg} \text {. } \\
& =\text { cardiac output estimated by the Fick procedure. }
\end{aligned}
$$


TABLE 3 Per cent perfusion per unit ventilated lung volume at rest, during and after exercise: individual data for right lung

\begin{tabular}{|c|c|c|c|c|c|c|c|c|c|c|c|c|c|c|c|}
\hline Case No. & $I$ & 2 & 3 & 4 & 5 & 6 & 7 & 8 & 9 & 10 & $I I$ & 12 & 13 & $I 4$ & 15 \\
\hline $\begin{array}{l}\text { Apex } \quad 5 \mathrm{~cm} \\
\text { Rest } \\
\text { Exercise } \\
2 \mathrm{~min} \text { after } \\
\text { I5 min after }\end{array}$ & $\begin{array}{r}9.6 \\
13.1 \\
10.9 \\
7.5\end{array}$ & $\begin{array}{l}5 \cdot 6 \\
7 \cdot 9 \\
7 \cdot 7 \\
8 \cdot 7\end{array}$ & $\begin{array}{r}8 \cdot 8 \\
6 \cdot 7 \\
9 \cdot 8 \\
12 \cdot 8\end{array}$ & $\begin{array}{r}\text { II.5 } \\
10 \cdot 9 \\
8 \cdot 4 \\
6 \cdot 9\end{array}$ & $\begin{array}{r}9 \cdot 2 \\
11 \cdot 9 \\
7 \cdot 4 \\
8 \cdot 1\end{array}$ & $\begin{array}{l}15 \cdot 6 \\
16 \cdot 2 \\
-\end{array}$ & $\begin{array}{l}17 \cdot 0 \\
16 \cdot 8 \\
19 \cdot 6 \\
13 \cdot 5\end{array}$ & $\begin{array}{l}15 \cdot 4 \\
17 \cdot 6 \\
\frac{1}{14} \cdot 6\end{array}$ & $\begin{array}{r}11 \cdot 2 \\
10 \cdot 7 \\
11 \cdot 4 \\
9 \cdot 9\end{array}$ & $\begin{array}{r}12 \cdot 5 \\
11 \cdot 0 \\
11 \cdot 8 \\
7 \cdot 8\end{array}$ & $\begin{array}{l}\text { II.4 } \\
14 \cdot 1 \\
16 \cdot 4 \\
15 \cdot 0\end{array}$ & $\begin{array}{l}8 \cdot 4 \\
7 \cdot 7 \\
8 \cdot 6 \\
8 \cdot 3\end{array}$ & $\begin{array}{l}10 \cdot 5 \\
10 \cdot 9 \\
13 \cdot 2 \\
12 \cdot 1\end{array}$ & $\begin{array}{l}\text { II } \cdot 2 \\
\text { II } 2 \\
-9 \cdot 5\end{array}$ & $\frac{-}{-}$ \\
\hline $\begin{array}{l}\text { Rest } \quad 10 \mathrm{~cm} \\
\text { Exercise } \\
2 \mathrm{~min} \text { after } \\
15 \mathrm{~min} \text { after }\end{array}$ & $\begin{array}{l}12.7 \\
13.7 \\
13.5 \\
11.6\end{array}$ & $\begin{array}{r}9 \cdot 4 \\
12 \cdot 2 \\
11 \cdot 7 \\
11 \cdot 7\end{array}$ & $\begin{array}{l}12 \cdot 7 \\
11 \cdot 7 \\
13 \cdot 1 \\
13 \cdot 5\end{array}$ & $\begin{array}{l}7 \cdot 6 \\
9 \cdot 3 \\
9 \cdot 3 \\
7 \cdot 7\end{array}$ & $\begin{array}{l}13.4 \\
15.3 \\
10.5 \\
12.6\end{array}$ & $\begin{array}{l}16 \cdot 6 \\
16 \cdot 4 \\
-\end{array}$ & $\begin{array}{l}10 \cdot 7 \\
11 \cdot 2 \\
12 \cdot 8 \\
12 \cdot 9\end{array}$ & $\begin{array}{l}\text { II } 0 \\
I I \cdot 2 \\
\frac{6}{6} \cdot 4\end{array}$ & $\begin{array}{l}18.5 \\
16.0 \\
14.9 \\
14.3\end{array}$ & $\begin{array}{l}11 \cdot 5 \\
12 \cdot 2 \\
10 \cdot 8 \\
11 \cdot 0\end{array}$ & $\begin{array}{l}11 \cdot 9 \\
13 \cdot 0 \\
12 \cdot 6 \\
11 \cdot 6\end{array}$ & $\begin{array}{l}14 \cdot 4 \\
14 \cdot 1 \\
15 \cdot 1 \\
14 \cdot 0\end{array}$ & $\begin{array}{l}12 \cdot 0 \\
12 \cdot 4 \\
12 \cdot 9 \\
13 \cdot 0\end{array}$ & $\begin{array}{l}17 \cdot 9 \\
17 \cdot 8 \\
14.6\end{array}$ & $\begin{array}{r}6.5 \\
10.5 \\
5.8 \\
6.1\end{array}$ \\
\hline $\begin{array}{l}\text { Rest } \quad 15 \mathrm{~cm} \\
\text { Exercise } \\
\quad 2 \text { min after } \\
15 \text { min after }\end{array}$ & $\begin{array}{l}15 \cdot 4 \\
12 \cdot 2 \\
14.3 \\
14.5\end{array}$ & $\begin{array}{l}15 \cdot 2 \\
14.5 \\
12 \cdot 7 \\
13.5\end{array}$ & $\begin{array}{l}14 \cdot 1 \\
13 \cdot 7 \\
14 \cdot 9 \\
13 \cdot 3\end{array}$ & $\begin{array}{r}9 \cdot 9 \\
9 \cdot 2 \\
10 \cdot 0 \\
9 \cdot 3\end{array}$ & $\begin{array}{l}14 \cdot 8 \\
14 \cdot 1 \\
15 \cdot 6 \\
16 \cdot 0\end{array}$ & $\begin{array}{l}11 \cdot 2 \\
13 \cdot 0 \\
-\end{array}$ & $\begin{array}{r}8 \cdot 4 \\
8 \cdot 1 \\
8 \cdot 6 \\
13 \cdot 5\end{array}$ & $\begin{array}{l}9 \cdot 4 \\
10 \cdot 9 \\
- \\
11 \cdot 4\end{array}$ & $\begin{array}{l}17 \cdot 3 \\
16 \cdot 9 \\
18 \cdot 6 \\
18 \cdot 2\end{array}$ & $\begin{array}{l}10 \cdot 2 \\
11 \cdot 0 \\
10 \cdot 6 \\
10 \cdot 6\end{array}$ & $\begin{array}{r}11 \cdot 9 \\
10 \cdot 4 \\
9 \cdot 9 \\
10 \cdot 5\end{array}$ & $\begin{array}{r}9 \cdot 9 \\
11 \cdot 4 \\
10 \cdot 2 \\
10 \cdot 3\end{array}$ & $\begin{array}{l}15.8 \\
13.6 \\
13.6 \\
13.9\end{array}$ & $\begin{array}{l}15 \cdot 3 \\
15 \cdot 7 \\
\frac{14.9}{14}\end{array}$ & $\begin{array}{l}12 \cdot 2 \\
12 \cdot 7 \\
10 \cdot 7 \\
12 \cdot 3\end{array}$ \\
\hline $\begin{array}{l}\text { Rest } 20 \mathrm{~cm} \\
\text { Exercise } \\
\quad 2 \mathrm{~min} \text { after } \\
\text { I } 5 \text { min after }\end{array}$ & $\begin{array}{l}15 \cdot 2 \\
12 \cdot 4 \\
14 \cdot 3 \\
17 \cdot 8\end{array}$ & $\begin{array}{l}19.6 \\
15.6 \\
15.0 \\
15.6\end{array}$ & $\begin{array}{l}13 \cdot 9 \\
12 \cdot 6 \\
12 \cdot 0 \\
12 \cdot 9\end{array}$ & $\begin{array}{l}12 \cdot 0 \\
10 \cdot 2 \\
12 \cdot 6 \\
10 \cdot 9\end{array}$ & $\begin{array}{l}15 \cdot 1 \\
13 \cdot 4 \\
17 \cdot 0 \\
18 \cdot 9\end{array}$ & $\begin{array}{l}4 \cdot 2 \\
5 \cdot 8 \\
-\end{array}$ & $\begin{array}{r}3 \cdot 9 \\
5 \cdot 3 \\
3 \cdot 5 \\
12 \cdot 0\end{array}$ & $\begin{array}{l}4 \cdot 6 \\
6 \cdot 7 \\
-11 \cdot 1\end{array}$ & $\begin{array}{r}7 \cdot 6 \\
12 \cdot 4 \\
12 \cdot 3 \\
12 \cdot 0\end{array}$ & $\begin{array}{r}9 \cdot 0 \\
9 \cdot 1 \\
8 \cdot 5 \\
12 \cdot 9\end{array}$ & $\begin{array}{r}10 \cdot 2 \\
8 \cdot 8 \\
8 \cdot 0 \\
10 \cdot 1\end{array}$ & $\begin{array}{l}10 \cdot 5 \\
10 \cdot 0 \\
10 \cdot 6 \\
10 \cdot 7\end{array}$ & $\begin{array}{l}14 \cdot 4 \\
12 \cdot 8 \\
11 \cdot 5 \\
13 \cdot 2\end{array}$ & $\begin{array}{l}12 \cdot 0 \\
11 \cdot 8 \\
\frac{12}{12 \cdot 4}\end{array}$ & $\begin{array}{r}12.9 \\
9.9 \\
12.6 \\
12.5\end{array}$ \\
\hline $\begin{array}{l}\text { Base } \quad 25 \mathrm{~cm} \\
\text { Rest } \\
\text { Exercise } \\
2 \text { min after } \\
15 \text { min after }\end{array}$ & & & & $\begin{array}{l}10 \cdot 2 \\
10.8 \\
12.5 \\
12.6\end{array}$ & & & & $\begin{array}{l}3 \cdot 1 \\
4 \cdot 6 \\
8 \cdot 7\end{array}$ & & $\begin{array}{r}7 \cdot 2 \\
9 \cdot 3 \\
7 \cdot 8 \\
10 \cdot 0\end{array}$ & $\begin{array}{l}7 \cdot 5 \\
7 \cdot 3 \\
5 \cdot 0 \\
6 \cdot 9\end{array}$ & & & & $\begin{array}{r}9 \cdot 1 \\
8 \cdot 1 \\
10 \cdot 3 \\
8 \cdot 6\end{array}$ \\
\hline
\end{tabular}

TABLE 4 Per cent perfusion per unit ventilated lung volume at rest, during and after exercise: individual data for left lung

\begin{tabular}{|c|c|c|c|c|c|c|c|c|c|c|c|c|c|c|c|}
\hline Case No. & $I$ & 2 & 3 & 4 & 5 & 6 & 7 & 8 & 9 & 10 & $I I$ & 12 & 13 & 14 & 15 \\
\hline $\begin{array}{l}\text { Apex } \quad 5 \mathrm{~cm} \\
\text { Rest } \\
\text { Exercise } \\
2 \mathrm{~min} \text { after } \\
15 \mathrm{~min} \text { after }\end{array}$ & $\begin{array}{r}8.5 \\
11.5 \\
9.0 \\
5.8\end{array}$ & $\begin{array}{r}5.2 \\
8.0 \\
10.0 \\
8 \cdot 1\end{array}$ & $\begin{array}{r}8 \cdot 2 \\
8 \cdot 5 \\
9 \cdot 0 \\
11 \cdot 3\end{array}$ & $\begin{array}{l}8 \cdot 4 \\
8 \cdot 2 \\
6 \cdot 1 \\
6 \cdot 9\end{array}$ & $\begin{array}{l}9 \cdot 2 \\
9 \cdot 3 \\
7 \cdot 8 \\
6 \cdot 7\end{array}$ & $\begin{array}{l}12 \cdot 4 \\
12 \cdot 7 \\
- \\
-\end{array}$ & $\begin{array}{l}18 \cdot 7 \\
18 \cdot 4 \\
20 \cdot 1 \\
12 \cdot 3\end{array}$ & $\begin{array}{l}16.1 \\
11.5 \\
- \\
12.5\end{array}$ & $\begin{array}{l}9 \cdot 4 \\
8 \cdot 4 \\
7 \cdot 6 \\
7 \cdot 3\end{array}$ & $\begin{array}{r}12.3 \\
10.2 \\
12.4 \\
5.6\end{array}$ & $\begin{array}{l}10.4 \\
11.5 \\
15.9 \\
10.1\end{array}$ & $\begin{array}{r}9.6 \\
10.0 \\
12.7 \\
12.0\end{array}$ & $\begin{array}{l}10.2 \\
13.1 \\
13.2 \\
10.5\end{array}$ & $\begin{array}{l}7 \cdot 2 \\
6.6 \\
8.5\end{array}$ & $\begin{array}{l}5 \cdot 0 \\
9 \cdot 2 \\
5 \cdot 4 \\
4 \cdot 0\end{array}$ \\
\hline $\begin{array}{l}\text { Rest } \quad 10 \mathrm{~cm} \\
\text { Exercise } \\
2 \mathrm{~min} \text { after } \\
15 \mathrm{~min} \text { after }\end{array}$ & $\begin{array}{l}11 \cdot 6 \\
13.3 \\
12.0 \\
12.9\end{array}$ & $\begin{array}{l}10.7 \\
13.9 \\
14.3 \\
12.6\end{array}$ & $\begin{array}{l}12.4 \\
13.2 \\
12.8 \\
12.6\end{array}$ & $\begin{array}{r}8.9 \\
10.5 \\
10.0 \\
9.0\end{array}$ & $\begin{array}{l}12 \cdot 2 \\
12.5 \\
12 \cdot 7 \\
10.4\end{array}$ & $\begin{array}{l}14.3 \\
12.8 \\
- \\
-\end{array}$ & $\begin{array}{l}16.5 \\
16.6 \\
15.3 \\
10.9\end{array}$ & $\begin{array}{l}13.6 \\
10.8 \\
-10.4\end{array}$ & $\begin{array}{l}17 \cdot 3 \\
14.2 \\
13.6 \\
13.1\end{array}$ & $\begin{array}{r}11 \cdot 0 \\
10.5 \\
11.5 \\
8.4\end{array}$ & $\begin{array}{r}10.7 \\
10.5 \\
10.8 \\
9.4\end{array}$ & $\begin{array}{l}15.3 \\
15.9 \\
14.7 \\
14.5\end{array}$ & $\begin{array}{l}13.3 \\
14.5 \\
13.3 \\
13.8\end{array}$ & $\begin{array}{l}11 \cdot 5 \\
12.6 \\
- \\
12.9\end{array}$ & $\begin{array}{r}8 \cdot 1 \\
12 \cdot 7 \\
8 \cdot 0 \\
8 \cdot 1\end{array}$ \\
\hline $\begin{array}{l}\text { Rest } \quad I 5 \mathrm{~cm} \\
\text { Exercise } \\
2 \mathrm{~min} \text { after } \\
15 \mathrm{~min} \text { after }\end{array}$ & $\begin{array}{l}13.9 \\
12.1 \\
12.8 \\
14.9 \\
\end{array}$ & $\begin{array}{l}16.4 \\
15.4 \\
15.7 \\
15.6 \\
\end{array}$ & $\begin{array}{l}15.9 \\
18.3 \\
14.7 \\
12.0\end{array}$ & $\begin{array}{r}10.2 \\
10.9 \\
9.8 \\
11.0\end{array}$ & $\begin{array}{l}\mathbf{1 3 . 1} \\
\mathbf{1 2 . 7} \\
\mathbf{1 4 . 7} \\
\mathbf{1 3 . 3} \\
\end{array}$ & $\begin{array}{l}14.7 \\
12.2 \\
- \\
-\end{array}$ & $\begin{array}{l}14.5 \\
14.9 \\
13.6 \\
12.6\end{array}$ & $\begin{array}{l}11 \cdot 0 \\
10.3 \\
- \\
10.2\end{array}$ & $\begin{array}{l}13.7 \\
13.6 \\
14.1 \\
14.7 \\
\end{array}$ & $\begin{array}{r}10.5 \\
9.6 \\
10.1 \\
11.0\end{array}$ & $\begin{array}{l}11 \cdot 2 \\
10.5 \\
10.0 \\
10.5 \\
\end{array}$ & $\begin{array}{l}16 \cdot 9 \\
16.8 \\
15 \cdot 1 \\
15.7 \\
\end{array}$ & $\begin{array}{l}\text { I1 } \cdot 9 \\
\text { I2.5 } \\
\text { I2.1 } \\
\text { I2.0 }\end{array}$ & $\begin{array}{l}12.8 \\
12.8 \\
\overline{13} \cdot 8\end{array}$ & $\begin{array}{l}12.8 \\
12.7 \\
12.9 \\
13.3 \\
\end{array}$ \\
\hline $\begin{array}{l}\text { Rest } 20 \mathrm{~cm} \\
\text { Exercise } \\
2 \text { min after } \\
\text { 15 min after }\end{array}$ & $\begin{array}{l}13.2 \\
11.6 \\
13.2 \\
14.9 \\
\end{array}$ & $\begin{array}{l}18.2 \\
12.7 \\
13.0 \\
14.4\end{array}$ & $\begin{array}{l}14 \cdot 1 \\
15 \cdot 6 \\
13 \cdot 8 \\
11 \cdot 7\end{array}$ & $\begin{array}{l}10.5 \\
10.9 \\
10.8 \\
13.2\end{array}$ & $\begin{array}{l}13 \cdot 0 \\
10 \cdot 9 \\
14 \cdot 5 \\
14 \cdot 0 \\
\end{array}$ & $\begin{array}{l}\text { II } \cdot 3 \\
\text { II I I } \\
- \\
-\end{array}$ & $\begin{array}{r}10.5 \\
8.8 \\
6.6 \\
12.3\end{array}$ & $\begin{array}{l}10.5 \\
10.2 \\
\text { II.9 }\end{array}$ & $\begin{array}{r}5 \cdot 0 \\
8.0 \\
7.6 \\
10.5 \\
\end{array}$ & $\begin{array}{r}8 \cdot 6 \\
9 \cdot 0 \\
8 \cdot 1 \\
11 \cdot 6\end{array}$ & $\begin{array}{l}9 \cdot 2 \\
7 \cdot 6 \\
8 \cdot 0 \\
9 \cdot 4\end{array}$ & $\begin{array}{l}15 \cdot 1 \\
14.1 \\
13.1 \\
14.5\end{array}$ & $\begin{array}{l}12.2 \\
10.6 \\
10.2 \\
11.4\end{array}$ & $\begin{array}{l}12.4 \\
\mathrm{II} \cdot 4 \\
\frac{13.4}{1}\end{array}$ & $\begin{array}{l}19.3 \\
13.8 \\
18.5 \\
20.0\end{array}$ \\
\hline $\begin{array}{l}\text { Base } \quad 25 \mathrm{~cm} \\
\text { Rest } \\
\text { Exercise } \\
2 \mathrm{~min} \text { after } \\
15 \mathrm{~min} \text { after }\end{array}$ & & & & $\begin{array}{r}10.0 \\
9.5 \\
11.4 \\
12.6\end{array}$ & & & & $\begin{array}{l}5 \cdot 5 \\
6 \cdot 4 \\
-7 \cdot 1\end{array}$ & & $\begin{array}{r}7 \cdot 4 \\
8 \cdot 5 \\
8 \cdot 3 \\
11 \cdot 4\end{array}$ & $\begin{array}{l}6 \cdot 0 \\
6 \cdot 2 \\
4 \cdot 4 \\
6 \cdot 4\end{array}$ & & & & $\begin{array}{l}14.4 \\
10.8 \\
15.0 \\
15.2\end{array}$ \\
\hline
\end{tabular}


No statistically significant differences between the right and left lung in any region or at any period (rest, exercise, $2 \mathrm{~min}$, and 15 min after exercise) were found. Therefore, in the statistical analysis we used the individual lungs. However, we must admit here that in some cases differences in basal perfusion between the left and the right lungs could be observed.

Our main interest was focused on the pulmonary blood flow in the basal areas. Two methods of expressing this basal flow were used, either the percentage of the totally registered activity per unit ventilated lung, or the perfusion gradient, calculated as the ratio between the basal and the apical perfusion. With regard to the resting values, the material was divided into different groups (Table 5).

Effect of exercise In those cases where the "perfusion gradient was higher than I (e.g. basal areas being better perfused than the apical ones), a significant decrease in the relative perfusion of the basal areas during exercise was found (or increase in apical ones).

When the perfusion gradient was lower than I (e.g. basal areas being less perfused ' than the apical ones), a significant increase in the relative perfusion of the basal areas during exercise or decrease in the apical ones was observed. When evaluating individually the perfusion data in the basal areas, different behaviour during exercise was found according to the initial level of basal blood flow. When the basal blood flow was ro per cent or more, a significant decrease in percentage - perfusion during exercise was found, while if the basal blood flow was lower than io per cent a significant increase in percentage perfusion was found.

Changes after exercise The basal blood flow at rest and 15 minutes after exercise was „compared. There was no significant difference in those lungs which had a perfusion gradient higher than 1 . However, in the lungs having perfusion gradients less than $I$ at rest there was a significant increase in the relative basal perfusion.

When the basal blood flow was Io per cent or more at rest no change occurred after exercise. However, if the basal blood flow was less than Io per cent, a significant percentage increase in flow after exercise was observed.

The mean values of mean pressures in the left atrium, the pulmonary artery, and the vascular resistance in the two groups of patients with basal flow more or less than Io per cent at rest are shown in Table 6. Patients with a basal blood flow less than ro per cent had a significantly higher mean pressure in the left atrium compared with those having a basal perfusion of more than Io per cent.

\section{Discussion}

We did not measure the amount of ${ }^{133}$ Xenon in the spirometer during equilibration nor did we measure the cardiac output by any other method. As a consequence only relative data on perfusion (in percentage of the totally registered perfusion per unit of ventilated lung volume) are given. The breath holding period must necessarily be short when making the measurements during or immediately after exercise. As a consequence we did not study the patients when performing maximal exercise. Effort was made to minimize the error due to the change in geometry during exercise.

A further source of error might be a change in alveolar volume during exercise. A changed position of the diaphragm could occur, which would influence the values and increase the alveolar volume particularly in the basal areas. There are, however, no studies available which show a definite change in alveolar volume

TABLE $5{ }^{133} X e$-regional perfusion per unit ventilated lung volume (per cent): Mean values, standard errors of mean, and $t$ values for differences ( $n=$ number of lungs studied)

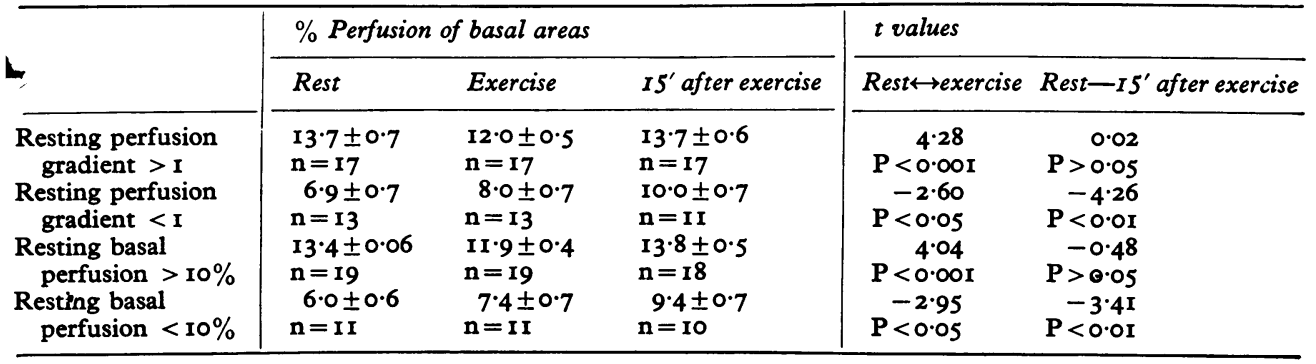


during exercise compared with resting condition in patients with mitral stenosis.

As we noticed a changing pattern of the lung perfusion with time during sitting in healthy subjects in our previous study (Bake et al., 1968), the resting period was standardized and only values of perfusion at 30 and 35 minutes of rest were used. Mean values of these two determinations have been used.

Our results, as summarized in Table 5, show that patients with mitral stenosis with a distribution of pulmonary blood flow at rest similar to that in normal subjects react in a predicted way during and after exercise (Bake et al., 1968). The relative basal flow decreases with exercise and returns to pre-exercise values after 15 minutes of rest. On the other hand, those patients with mitral stenosis who at rest have a decrease in the relative basal flow increase this flow during exercise. Furthermore, this exercise-induced increase persists after 15 minutes of rest. Whether the material was divided according to the percentage basal flow or to the perfusion gradient as earlier defined, the same difference in behaviour was found.

However, it must be stated that in individual patients with a low perfusion gradient at rest a decrease occurs with exercise, as seen in Case II. This patient had a conspicuous increase in pulmonary vascular resistance during exercise at catheterization. It may be implied that in this patient anatomical changes were mainly responsible for the increase in pulmonary vascular resistance.

In 3 patients (Cases 6, 7, and 8) differences between the left and right side in the basal pulmonary blood flow at rest were found. Comparing the basal blood flow in these subjects 15 minutes after exercise with the resting values, we found equalized basal flows on the two sides after exercise in spite of the obvious difference at rest. Thus, a side difference in the perfusion at rest, which could be caused by pulmonary embolism, disappeared after exercise. These findings may imply that studies of the distribution of perfusion should be performed not only at rest but possibly also during and after exercise.

Pulmonary circulation in mitral stenosis is characterized by passive pulmonary hypertension which is due to the obstruction of the mitral valve. Some patients with mitral stenosis show, however, an increased pulmonary vascular resistance. This increase can be due either to anatomical changes in the small vessels as described by Parker and Weiss (1936), or to functional vasoconstriction (Söderholm and Werkö, 1959; Söderholm, Werkö, and Widimský, 1962; West et al.,
TABLE 6 Mean pressures in left atrium and pulmonary artery and pulmonary vascular resistance in patients with basal blood flow above and below Io per cent at rest: Mean values and $t$ values for differences

\begin{tabular}{|c|c|c|c|}
\hline & $\begin{array}{l}\text { Mean left atrial } \\
\text { pressure } \\
m m H g\end{array}$ & $\begin{array}{l}\text { Mean pulm. } \\
\text { artery pressure } \\
\text { mmHg }\end{array}$ & $\begin{array}{l}\text { Pulmonary } \\
\text { vascular } \\
\text { resistance } \\
\mathrm{mmHg}(\mathrm{l} . / \mathrm{min})\end{array}$ \\
\hline Basal flow $>10 \%$ at rest: & $\begin{array}{l}\text { I } 5 \pm 2 \\
n=8\end{array}$ & $\begin{array}{l}25 \pm 2 \\
n=7\end{array}$ & $\begin{array}{l}2 \cdot 8 \pm 0 \cdot 6 \\
n=7\end{array}$ \\
\hline Basal flow $<10 \%$ at rest: & $\begin{array}{l}22 \pm 3 \\
n=5 \\
t=2 \cdot 22 \\
P<0.05\end{array}$ & $\begin{array}{l}38 \pm 7 \\
n=5 \\
t=I \cdot 93 \\
P>0.05\end{array}$ & $\begin{array}{l}4 \cdot 7 \pm I \cdot 7 \\
n=5 \\
t=I \cdot 19 \\
P>0.05\end{array}$ \\
\hline
\end{tabular}

I965; Widimský et al., I969). The results of the latter investigation show that it is possible to decrease the pulmonary artery pressure and pulmonary vascular resistance both at rest and during exercise with the infusion of acetylcholine into the right heart. Studies on patients with mitral valvular disease after operation imply that an early postoperative decline in pulmonary vascular resistance can be found (Matloff et al., 1968).

Another mechanism of the increased pulmonary vascular resistance was described by West et al. (1965). They found in experiments on isolated lungs that an increase in pulmonary venous pressure induced an increase in perivascular fluid. According to West et al. this perivascular oedema can compress small pulmonary vessels, and thus increase the flow resistance. However, this theory has been recently criticized by Ritchie, Schauberger, and Staub (1969).

The increase in pulmonary vascular resistance in patients with mitral stenosis is not evenly distributed in the lungs. Steiner and Quinn (1968) demonstrated with the use of $x$-rays, that in patients with mitral stenosis the basal areas were less perfused than the apical ones. Similar findings have been observed with the use of angiographic techniques.

In healthy subjects in the sitting or standing position, the basal pulmonary areas are more perfused than the apical ones (Bake et al., 1968; Ball et al., 1962; Oppelt et al., I966; West and Dollery, 1960; West, 1962). This perfusion gradient between basal and apical areas can disappear or become even inverted in mitral stenosis (Dawson et al., 1965; Dollery and West, 1960; Oppelt et al., 1966). It was shown by one of us (Jebavý et al., 1970), that this inverse perfusion gradient is related to the pulmonary artery pressure or to the left 
atrial pressure or to the pulmonary vascular resistance.

In this material we found no significant correlation between percentage basal flow and either $\overline{\mathrm{P}}_{\mathrm{LA}}, \overline{\mathrm{P}}_{\mathrm{PA}}$, or PVR at rest. The only correlation between the findings at catheterization and the perfusion data is the one shown in Table 6: the group of patients with basal flow less than Io per cent at rest has a significantly higher $\overline{\mathrm{P}}_{\mathrm{LA}}$ than the group with a higher basal flow. However, the haemodynamic data were obtained separately (mostly within one week) from the study of the ${ }^{133}$ Xenon regional perfusion. The exercise during the catheterization procedure was performed in the supine position and during the ${ }^{133}$ Xenon studies in the sitting position, and the patients were studied at different levels of exercise during catheterization and during the ${ }^{133}$ Xenon studies.

With these reservations in mind, the data presented seem to imply that patients with a basal flow of more than ro per cent at rest have a haemodynamically less significant mitral stenosis than those with a basal flow less than ro per cent.

Our results could hypothetically be ex" plained by a dilatation of the basal lung vessels. If the West theory plays a significant role, then in patients with a low basal flow this flow would decrease during exercise because of increasing perivascular oedema due to a rising mean left atrial pressure. We observed, however, opposite findings in our patients. A decrease in pulmonary vascular resistance at the basal areas in these patients ..is assumed, which could imply vasodilatation.

No conclusions can be drawn from our investigation as to the mechanism of this vasodilatation. However, one may hypothetically assume that the exercise increases alveolar ventilation and thus alveolar oxygen tension in mitral stenosis patients, especially in the basal areas which are hypoventilated at rest as shown by Jebavý et al. (1970). Thus the Euler-Liljestrand vasoconstrictive effect (von Euler and Liljestrand, 1946) on the pulmonary circulation in the basal areas might be lessened by an increase in the alveolar oxygen tension during exercise, and this effect might last 25 minutes after exercise.

Another explanation of the vasodilatation could be stress relaxation of the pulmonary vessels as described by Sarnoff and Berglund (1952). The stress relaxation of the vessels at the basal areas might also explain the change still present 15 minutes after exercise.

We may conclude that patients with mitral valvular disease and a high relative pulmonary basal blood flow decrease this flow during exercise while those having low relative pulmonary basal blood flow increase pulmonary basal blood flow during exercise. This increased blood flow was still present 15 minutes after exercise. It is assumed that this change is due to vasodilatation during and after exercise in the basal fields.

\section{References}

Bake, B., Bjure, J., Grimby, G., Milic-Emili, J., and Nilsson, N. J. (1967). Regional distribution of inspired gas in supine man. Scandinavian fournal of Respiratory Diseases, 48, 189.

Bake, B., Bjure, J., and Widimský, J. (I968). The effect of sitting and graded exercise on the distribution of pulmonary blood flow in healthy subjects studied with the ${ }^{133}$ Xenon technique. Scandinavian fournal of Clinical and Laboratory Investigation, 22, 99.

Ball, W. C., Stewart, P. B., Newsham, L. G. S., and Bates, D. V. (1962). Regional pulmonary function studied with Xenon ${ }^{133}$. Fournal of Clinical Investigation, 41, 519.

Dawson, A., Kaneko, K., and McGregor, M. (1965). Regional lung function in patients with mitral stenosis studied with $\mathrm{Xenon}^{133}$ during air and oxygen breathing. Fournal of Clinical Investigation, 44, 999.

Dollery, C. T., and West, J. B. (1960). Regional uptake of radioactive oxygen, carbon monoxide and carbon dioxide in the lungs of patients with mitral stenosis. Circulation Research, 8, 765.

von Euler, U. S., and Liljestrand, G. (1946). Observations on pulmonary arterial blood pressure in the cat. Acta Physiologica Scandinavica, 12, 301.

Forsberg, S. A. (1964). Pulmonary blood volume in man. A study using the double indicator technique in patients with cardiovascular disease. Acta Medica Scandinavica, 175, Suppl. 410.

Jebavý, P., Runczik, I., Oppelt, A., Tilsch, J., Staněk, V., and Widimský, J. (1970). Regional pulmonary function in patients with mitral stenosis in relation to haemodynamic data. British Heart fournal, 32, 330.

Matloff, J. M., Dalen, J. E., Exter, L., and Harken, D. E. (1968). Hemodynamic response to discoid mitral valve replacement. Circulation, 37 and $\mathbf{3 8}$, Suppl. 2, p. 94.

Oppelt, A., Widimský, J., Staněk, V., Bláha, V., Runczik, I., and Vavrejn, B. (I966). Regional pulmonary function in normals and in disease studied by intravenously injected ${ }^{13} \mathrm{Xe}$. Bulletin de PhysioPathologie Respiratoire, 2, 653.

Parker, F., Jr., and Weiss, S. (1936). The nature and significance of the structural changes in the lungs in mitral stenosis. American fournal of Pathology, 12, 573.

Paulin, S., and Varnauskas, E. (1962). Selective transseptal angiocardiography. Acta Radiologica, 57, 3.

Ritchie, B. C., Schauberger, G., and Staub, N. C. (1969). Inadequacy of perivascular edema hypothesis to account for distribution of pulmonary blood flow in lung edema. Circulation Research, 24, 807.

Sarnoff, S. J., and Berglund, E. (1952). Pressurevolume characteristics and stress relaxation in the pulmonary vascular bed of the dog. American fournal of Physiology, 171, 238.

Söderholm, B., and Werkö, L. (1959). Acetylcholine and the pulmonary circulation in mitral valvular disease. British Heart fournal, 21, I. 
Söderholm, B., Werkö, L., and Widimský, J. (1962). The effect of acetylcholine on pulmonary circulation and gas exchange in cases of mitral stenosis. Acta Medica Scandinavica, 172, 95.

Steiner, S. H., and Quinn, J. M. (r968). Cardiovascular hemodynamics; determination from the distribution of pulmonary blood flow in seated patients. fournal of the American Medical Association, 203, 850.

West, J. B. (1962). Regional differences in gas exchange in the lung of erect man. Fournal of Applied Physiology, 17, 893 .
West, J. B., and Dollery, C. T. (1960). Distribution of blood flow and ventilation-perfusion ratio in the lung, measured with radioactive $\mathrm{CO}_{2}$. Fournal of Applied Physiology, 15, 405.

West, J. B., Dollery, C. T., and Heard, B. E. (1965). Increased pulmonary vascular resistance in the dependent zone of the isolated dog lung caused by perivascular edema. Circulation Research, 17, 191.

Widimský, J., Hurych, J., Staněk, V., and Kasalický, J. (I969). Effect of unilateral pulmonary artery occlusion on pulmonary circulation in patients with pulmonary hypertension in mitral stenosis. British Heart fournal, 31, 172. 